\title{
La vertu de l'instrument. À propos de quelques inscriptions gravées sur des qin anciens
}

\section{Georges Goormaghtigh}

\section{(2) OpenEdition \\ 12 Journals}

Édition électronique

URL : https://journals.openedition.org/ethnomusicologie/1385

ISSN : 2235-7688

\section{Éditeur}

ADEM - Ateliers d'ethnomusicologie

Édition imprimée

Date de publication : 31 décembre 1994

Pagination : 95-103

ISBN : 2-8257-0503-9

ISSN : $1662-372 X$

\section{Référence électronique}

Georges Goormaghtigh, «La vertu de l'instrument. À propos de quelques inscriptions gravées sur des qin anciens », Cahiers d'ethnomusicologie [En ligne], 7 | 1994, mis en ligne le 03 janvier 2012, consulté le 21 septembre 2021. URL : http://journals.openedition.org/ethnomusicologie/1385

Ce document a été généré automatiquement le 21 septembre 2021.

Tous droits réservés 


\title{
La vertu de l'instrument. À propos de quelques inscriptions gravées sur des qin anciens
}

\author{
Georges Goormaghtigh
}

Wen, le maître de musique du pays de Zheng avait
joué de la cithare toute la journée ; dans un élan
d'enthousiasme il se leva et se prosterna par deux
fois devant son instrument en disant : «J'ai tout à
apprendre de toi. Comme toi, je voudrais être
inépuisable ! »
Lüshi chunqiu ${ }^{1}$

1 La Renaissance qui vit l'essor de l'humanisme connut parallèlement un formidable développement dans le domaine de la lutherie et dans celui de la facture d'instruments à clavier. On retrouve aujourd'hui encore des témoignages de l'esprit de cette époque dans les formules grecques ou latines inscrites sur certains instruments du XVI siècle : «Le chant est pour l'homme le médecin de la douleur " peut-on lire sur la lira da bracio de Giovanni d'Andrea datée de 1511, conservée au Kunsthistorisches Museum de Vienne. Hans Ruckers l'Ancien reprend la même idée dans une inscription peinte sous les deux claviers d'un virginal de 1581 qu'on peut admirer au Metropolitan Museum of Art de New York: «La musique est le remède le plus doux contre toutes les peines. » Plus originale est l'inscription figurant sur une épinette vénitienne de 1540 conservée dans le même musée : «Je suis riche d'or et riche de sons, ne me joue pas si tu n'es pas bon. » Sur un instrument plus tardif, on peut encore lire: «Vivant j'étais silencieux, mort je chante doucement. » Mentionnons finalement la belle et laconique formule qui orne un positif construit en 1724 par Silberman : «A la seule gloire de Dieu. » 
2 Quel musicien n'a pas rêvé de posséder un jour l'instrument idéal : un instrument dont les qualités d'émission exceptionnelles en feraient le médiateur parfait, le révélateur infaillible de son art? Ce rêve parfois se réalise et peut alors donner lieu à de grands moments musicaux. Rares, cependant, sont les instrumentistes qui laissent un témoignage écrit de telles rencontres, car il s'agit pour eux d'une expérience qui, par nature, se situe au-delà du langage. Certains lettrés chinois se sont pourtant livrés à ce redoutable exercice en rendant par écrit hommage aux vertus de leur instrument favori, le qin, antique cithare à sept cordes. Il faut dire que la culture des lettrés les prédisposait à s'attaquer à ce thème : une connaissance souvent profonde des lois de la calligraphie, de la peinture et de la musique associée à une formation poétique très poussée leur permettait d'improviser sur-le-champ de remarquables poèmes, aussi bien lors de joutes poétiques où le vin ne manquait pas, qu'à l'occasion de réunions musicales. C'est lors de telles rencontres que les connaisseurs pouvaient évaluer les mérites respectifs de certains qin précieux, comme les instruments construits par des membres de la famille Lei, grands luthiers de la dynastie des Tang (618-907). Ces remarques, souvent formulées en vers et consignées par écrit dans une calligraphie élégante, étaient parfois reportées sur l'instrument lui-même, exactement à la manière des textes gravés sur stèle pour proclamer la solennité, assurer la pérennité et permettre la reproduction par estampage de ces écrits.

3 La partie inférieure du qin, surface plane et lisse, offre un support idéal où graver des caractères. Il n'est pas étonnant qu'on ait été tenté d'y inscrire des textes et d'y apposer des sceaux, un peu comme les colophons qui ornent les peintures chinoises et en rehaussent le prestige ${ }^{2}$. 
Fig. 1 : Qin construit en 1637 par le Prince de Lu.
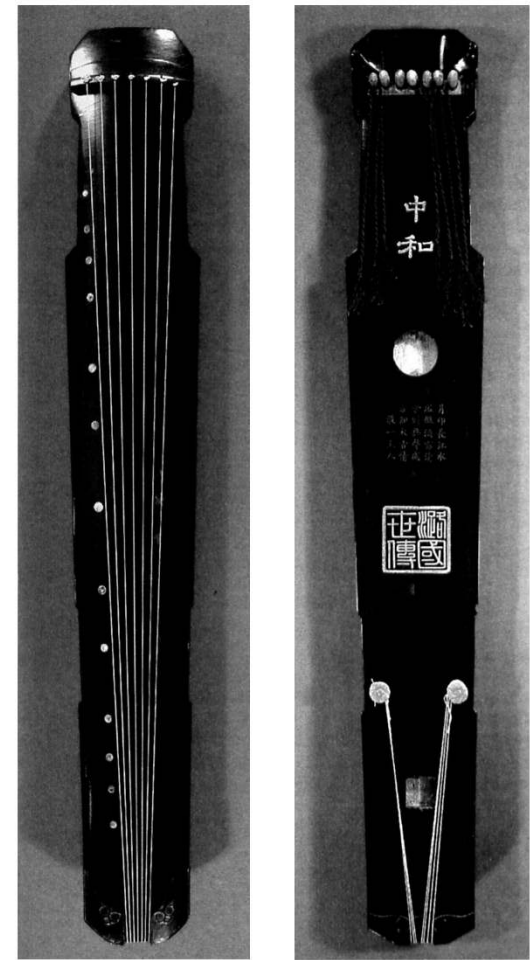

La caisse de résonance en bois de paulownia (partie supérieure) et de catalpa (partie inférieure) est entièrement laquée. Les pieds et les chevilles en jade vert retiennent sept cordes de soie.

Le nom de l'instrument, Zhonghe « Harmonie du Milieu », est gravé dans la laque en grands caractères de chancellerie.

Sous l'ouïe circulaire (symbolisant le Ciel) est inscrit un poème suivi d'un sceau (voir fig. 2) 
Fig. 2 : Poème inscrit dans la laque du qin « Harmonie du Milieu » :

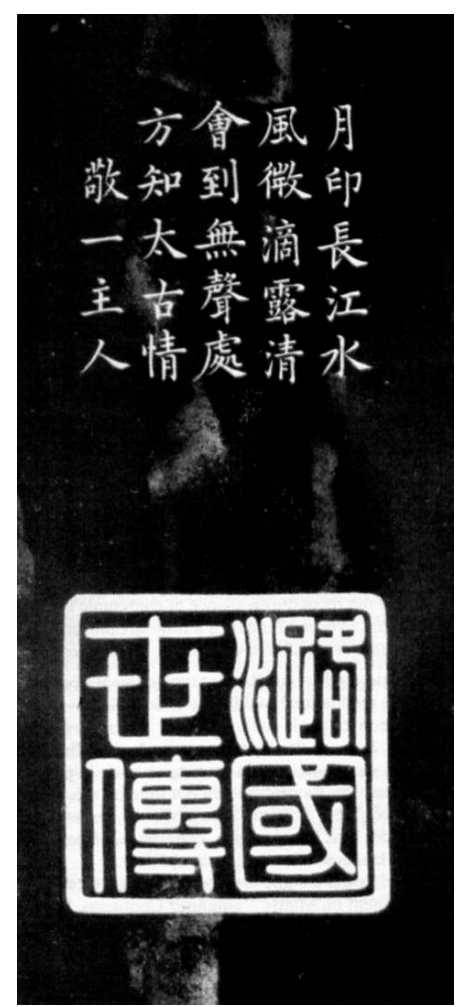

La lune se reflète sur l'eau du Fleuve bleu

Une brise douce circule, des gouttes de rosée rutilent.

Qui comprend le sens du silence

Peut connaitre l'esprit de la haute Antiquité.

Signé : « Le maître qui vénère l'Un », nom littéraire du Prince de Lu.

Le grand sceau carré gravé sous le poème signifie : «Transmis de génération en génération dans la principauté de Lu ».

Certaines de ces inscriptions ont été rassemblées et sont conservées dans un gros ouvrage publié en 1590, le Qinshu daquan, véritable somme encyclopédique sur le qin ${ }^{3}$. Le plus ancien de ces textes remonte au début du deuxième siècle de notre ère ; il est de la main du poète Li You (55-135) des Han orientaux et reflète bien la tournure morale que prend volontiers la réflexion sur la musique à cette époque :

«Le son du qin purifie le cœur de ses penchants mauvais, mais il émeut aussi celui dont la nature est droite. Ne retenant que les mélodies les plus nobles, il écarte les sonorités lascives et empêche les excès. Sa musique pénétrante, harmonieuse et correcte emplit nos cœurs de joie mais jamais ne déborde. »

Une idée importante de ce texte est la vertu purificatrice de la musique du qin, thème que l'on retrouve souvent par la suite dans la littérature chinoise, entre autre sous le pinceau du grand poète Li Bai (701-762). Dans un huitain célèbre, ce dernier compare la musique de qin au bruissement du vent dans les pins et aux flots d'une rivière qui lui «purifient l'esprit $»^{4}$. La pratique du qin a d'ailleurs toujours été conçue par les lettrés comme un moyen de perfectionnement personnel. « L'homme de bien ne joue pas du qin sans raison, car c'est par lui qu'il cultive sa nature et harmonise ses émotions " peut-on lire dans un manuel de qin qui reprend une idée chère aux penseurs de l'antiquité 5 . L'auteur de notre inscription fait encore écho à une brève définition du qin donnée par l'historien Ban Gu (32-92) : "Qin signifie interdire, (jeu de mots sur l'assonance de qin "cithare" et jin 
"interdire") ; en interdisant les passions licencieuses, on rectifie le cœur des hommes ${ }^{6}$. Mais Li You emprunte aussi à un texte plus ancien, le Mémoire sur la musique (III ${ }^{\mathrm{e}}$ siècle av. J.C.), les adjectifs « pénétrant » et « harmonieux » pour décrire les qualités esthétiques de la musique de qin, sans lesquelles celle-ci ne saurait avoir d'efficacité. Dans cette tradition de pensée, les domaines de l'éthique et de l'esthétique sont, dès l'origine, intimement liés : de même que l'homme se révèle à travers sa musique, c'est la pratique de son art qui va lui permettre de réaliser pleinement son humanité.

De nombreuses inscriptions recueillies dans le Qinshu daquan datent de la dynastie des Song (960-1275), époque de grand essor dans tous les domaines de la vie en Chine. La lutherie n'échappe pas à la règle avec, entre autre, la rédaction de plusieurs traités sur la construction du qin. Cet intérêt pour l'aspect le plus concret de l'instrument, dont dépendent les qualités subtiles du timbre, transparaît dans l'inscription qu'un lettré des Song du Nord, Huang Tingjian (1045-1105), grand poète féru de bouddhisme et admirable calligraphe, fit graver sur un qin:

"Quand le bois de la table d'harmonie est trop mince, le son a tendance à se disperser, quand il est trop épais, la résonance devient courte comme celle de la pierre ; si l'instrument est trop volumineux, le son est criard ; trop étroit, il n'a pas d'étendue et trop long, il vibre de façon désagréable. Un instrument exempt de ces cinq défauts produira des sons toujours parfaitement en accord avec les échelles et ayant la plénitude de la cloche qui résonne.»

«La cloche qui résonne » est justement le nom du qin pour lequel Huang Tingjian écrivit ce commentaire ${ }^{7}$. Ici la cloche suggère naturellement l'idée de la puissance sonore, de la durée vibratoire et du caractère percussif de ses attaques, autant de qualités qui se retrouvent sous les doigts d'un grand musicien lorsqu'il dispose d'un bon instrument. Mais par-delà cet idéal sonore, le nom de ce qin évoque surtout un idéal musical, celui de l'antique musique cérémonielle des Zhou Occidentaux (1122-771 av.J.C.) tant admirée par Confucius. Le carillon y jouait un rôle prépondérant. Par allusion symbolique à cette musique vénérable, certains luthiers incorporaient parfois à la laque qui recouvre l'instrument une petite quantité de paillettes de bronze dont la présence était censée conférer au qin ce timbre idéal.

8 L'association du timbre du qin à celui de la cloche est du reste un thème que l'on retrouve en littérature, notamment dans le poème de Li Bai que nous avons déjà cité. Il décrit la musique que lui joue un moine du Mont Emei au Sichuan sur un qin ancien :

« Je crois entendre le bruissement des pins dans mille ravins.

Mon esprit est purifié par ces flots.

L'écho des dernières notes pénètre la cloche

couverte de givre... »

Le poète ne fait que suggérer en passant, mais avec force, l'idée que le son de ce qin est capable de faire vibrer par sympathie l'énorme cloche du monastère.

Avec les Song du Sud (1127-1279) les inscriptions reprennent un tour plus philosophique et sont cette fois teintées de néo-confucianisme. Ce courant de pensée, alors en plein développement, avait pour thème central l'identité de la nature humaine et de l'ordre universel. Les deux inscriptions suivantes sont de la main du célèbre Zhu Xi (1130-1200), philosophe connu pour sa brillante synthèse des idées néo-confucéennes. Pour lui, les qualités matérielles et symboliques de l'instrument se combinent et font du qin un modèle et un idéal de vertu :

«Cet instrument n'est fait ni de pierre ni de métal (allusion aux lithophones et aux carillons de l'Antiquité), il a pourtant toutes les vertus du jade. Quand le tonnerre 
reste caché en lui, son esprit languit ; mais, dès qu'on le fait sonner, c'est le retour du printemps sur toutes les créatures. Conscient de ce qui fait sa valeur, je le chéris comme un être vivant.

Pour m'améliorer, souvent je me recueille en silence et contemple le retour des choses à leur origine; mais, avec ma tendance à toujours ployer sous le poids des soucis, je suis encore loin d'égaler sa droiture et son aisance. »

11 Zhu Xi fait ici allusion à des pratiques de recueillement typiquement néo-confucéennes en partie héritées de la méditation bouddhique et du taoïsme - où tout le travail consiste à tenter de remonter, par la contemplation, à l'origine des phénomènes (voir Laozi chap. 16). Le nom de l'instrument sur lequel $\mathrm{Zhu} \mathrm{Xi}$ fit inscrire ce texte comporte deux caractères, fu « le retour » et zhai « la purification ", référence directe à cette ascèse. La pratique du qin joue un rôle central dans cet exercice. Sa musique, véritable dialogue avec le silence, en rendant le musicien attentif au lent retour des sons à leur origine, l'engage peu à peu à observer tous les phénomènes avec la même attention contemplative.

La deuxième inscription décrit le qin comme le vecteur privilégié de ce qu'il y a de plus vivant dans l'enseignement du Maître - entendez Confucius. Elle traduit l'émotion que peut ressentir le musicien devant un instrument exceptionnel :

«Quand je le touche, il est grave et profond comme si j'avais à affronter une passe périlleuse, mais dès que je me confie à lui, il tinte clairement ; il me suffit alors de guider vers la vallée cette source jaillissante.

On ne peut plus entendre les paroles du Maître, mais il a confié à cet instrument toute la pénétration de son esprit. Ainsi s'est-elle transmise jusqu'à nous. »

Rappelons que, parmi les nombreuses formes qu'a pu prendre le qin au cours des âges, le modèle de loin le plus courant s'appelle justement « Confucius » (Zhongni); l'instrument étant en quelque sorte l'incarnation du sage et de ses vertus.

On retrouve, dans une autre inscription de cette époque, la même admiration mêlée de révérence pour l'instrument ancien capable de révéler des domaines insoupçonnés à celui qui en joue, en lui ouvrant des espaces infinis. L'auteur, Wei Zongwu, un amateur de poésie intéressé, comme beaucoup de ses contemporains, aux aspects cosmologiques du qin, exprime ici son admiration pour un instrument remarquable qu'il avait nommé «Grande élégance » Daya, en référence, là encore, à la musique cérémonielle des Zhou :

«L'essence du Soleil, l'éclat de la Lune et l'harmonie du Ciel s'unissent pour produire cette sonorité consistante, pleine de vertu, ronde et limpide. Lorsque ces notes retentissent, leur timbre profond se prolonge, puis elles retournent au silence. S'accordant aux échelles, elles semblent faites pour jouer les airs vénérables de la haute Antiquité.

Quand je frappe légèrement le bois de cet instrument, il tinte comme du bronze ou $\mathrm{du}$ jade ; mais si je frappe plus fort, il se met à rugir comme un tigre ou un dragon.

C'est là bien autre chose qu'un instrument seulement agréable à jouer... »

Le qin construit par un grand luthier, avec un matériau exceptionnel, ne ressemble plus aux instruments ordinaires; ce n'est pas une simple amélioration qualitative qui se produit, mais un véritable changement de nature. Avec un tel instrument, le musicien peut faire l'expérience des dimensions cosmiques de sa musique, comme si, soudain, il était mis en présence des forces élémentaires. C'est là tout le sens de ce « rugissement du tigre et du dragon ». Ces sonorités naturelles et surnaturelles prennent le pas, - dès que l'instrument est véritablement sollicité - sur la musique humaine, symbolisées ici par le timbre du bronze et du jade ${ }^{8}$.

Nous terminerons par une autre inscription du philosophe $\mathrm{Zhu} \mathrm{Xi,} \mathrm{qui} \mathrm{donne} \mathrm{cette} \mathrm{fois} \mathrm{la}$ parole à son instrument et commence par lui faire prononcer deux phrases imprégnées 
de moralisme. Les termes sont presque textuellement ceux de notre première citation et témoignent de la pérennité de cette idée d'une dimension éthique dans la musique de qin. Mais le texte, très bref, se termine sur une ouverture : il évoque l'action du Ciel et de la Terre. En jouant, le musicien prend part à cette action, vaste mouvement dans lequel il entre et qui, aussitôt, le porte. Véritable microcosme, bombé sur le haut comme le Ciel et plat en dessous comme la Terre, l'instrument lui-même est à l'image de cet échange. Pour celui qui en joue, il est à la fois le lieu et le moyen de la révélation. C'est par lui que s'opère la transformation intérieure, que s'élabore et se concrétise l'expérience esthétique :

«Cultive la rectitude et l'harmonie qui t'habitent, Empêche les passions mauvaises de naître en ton cœur ;

Le Ciel et la Terre ne parlent pas, mais les êtres obéissent;

Moi seul, avec toi, pouvons en sonder toute la profondeur. »

\section{BIBLIOGRAPHIE}

VAN GULIK Robert H., 1969, The Lore of the Chinese Lute : an Essay in Ch'in Ideology. Tokyo, Rutland (Vermont) : Sophia University \& The C.E.Tuttle Cy. [A Monumenta Nipponica Monograph].

Li Bai quanji, 1977, Pékin : Zhonghua shuju.

Lüshi chunqiu jiaoshi, 1984, Shanghai : Xuelin chubanshe

Qinqu jicheng, 1981, Wenhuabu wenxue yishu yanjiuyuan Yinyue yanjiusuo Beijing guqin yanjiuhui. 24 vol. Pékin : Zhonghua shuju.

Zhongguo gudai yuelun xuanji, 1981, Wenhuabu wenxue yishu yanjiuyuan Yinyue yanjiusuo Beijing guqin yanjiuhui. Pékin : Renmin yinyue chubanshe.

\section{NOTES}

1. Lüshi chunqiu jiaoshi, p. 1050.

2. Les premiers textes gravés sur des instruments de musique en Chine datent de la fin du II millénaire avant notre ère. Ce sont des inscriptions sur cloches généralement courtes. Par la suite, ces textes prirent une ampleur considérable, comme le prouvent les fameuses cloches du carillon du Marquis Yi de Zeng ( $\mathrm{V}^{\mathrm{e}}$ siècle av. J.C.). Pour ce qui est des inscriptions sur qin, les plus anciennes ne semblent remonter qu'au deuxième siècle de notre ère. On trouvera une excellente analyse des différents types d'inscriptions figurant non seulement sur l'instrument mais aussi à l'intérieur du qin dans R. van Gulik pp. 197-200.

3. Voir Qinqu jicheng vol. V, pp. 400-403.

4. Li Bai quanji, vol. II, p. 1129.

5. Wang Fu, Lixuezhai qinpu in Zhongguo gudai yuelun xuanji, p. 414.

6. Ban gu, Baihu tong. "De lun. Liyue”, in Zhongguo gudai yuelun xuanji, p. 101.

7. En Chine les lettrés donnaient volontiers un nom à leur instrument: « Rugissement du tigre », " Neige sur les pins », « Tonnerre du printemps » etc. Ces noms, toujours très poétiques, sont 
répertoriés sous forme de liste dans différents manuels de qin. Pour une description de ces listes voir R. van Gulik, pp. 104-105.

8. Voici encore deux inscriptions extraites du même recueil que nous livrons au plaisir du lecteur:

\section{« Age d'or "}

Cette cithare est pure comme le Sans-Nom;

Je l'effleure, elle résonne et captive mon cœur;

Porteuse de silence elle n'est point muette;

Pleine de charme elle ne cherche pas à séduire.

Moi seul te connais.

Hautes montagnes, profondes eaux!

«Cloche givrée»

En lui vivent les sons vastes du Ciel et de la Terre,

D'avance il connaît toutes les transformations.

Le prunier va fleurir,

La lune, parcourir le ciel.

En haut le vide,

En bas l'horizon laiteux.

Mon cœur est pareil à ce disque de jade immaculé. Plus de qin, plus de cordes !

\section{RÉSUMÉS}

Dans la musique classique chinoise, les mots jouent un rôle considérable. Ce besoin de s'exprimer verbalement se retrouve jusque dans les inscriptions que les lettrés faisaient graver sur des qin anciens pour célébrer les vertus de certains instruments exceptionnels. Le Qinshu daquan, ouvrage encyclopédique sur le qin publié en 1590 rassemble une trentaine de ces inscriptions. Nous en avons traduit et analysé une sélection datant la plupart de la dynastie des Song (960-1275) car elles sont autant de témoignages sur les conceptions esthétiques d'une période particulièrement riche de l'histoire culturelle chinoise.

In classical chinese music words can play a considerable role. The urge for expression, the taste for writing, so typical of chinese literati, can be traced even to the inscriptions engraved on certain ancient qin. The Qinshu daquan, an encyclopaedic opus devoted to that instrument published in 1590, reproduces about thirty such inscriptions, which are often of considerable literary and musical interest. We believed it would be useful to translate and to analyse a selection of these texts, mainly dating from the Song dynasty (960-1275), because they offer first hand testimony on the musical thought of that crucial period in the development of chinese aesthetics.

\section{AUTEUR}

\section{GEORGES GOORMAGHTIGH}

Georges Goormaghtigh a vécu plusieurs années en Chine populaire et fait de nombreux séjours à Hong Kong et à Taïwan. Parallèlement à des études de littérature chinoise classique il entreprend 
dès 1973 l'apprentissage du qin auprès de Madame Tsar Teh-yun (Cai Deyun), maître de l'école Fanchuan, à Hong Kong. Il enseigne le chinois à l'Université de Genève et donne des cours de qin aux Ateliers d'ethnomusicologie. Il a publié L'Art du qin (Bruxelles 1990). 